\title{
The Effects of Packaging on Collision Energy Absorption of Automotive
}

\section{Seat Headrest}

\author{
Chen Minggang ${ }^{1,2, a}$, Zhou Lei ${ }^{3}$, Meng Zhenghua ${ }^{1, b^{*}}$, Wei Xuebing ${ }^{3}$, Wang Hui ${ }^{1}$ \\ ${ }^{1}$ Hubei Key Laboratory of Advanced Technology for Automotive Components, Wuhan University of Technology, Wuhan 430070, China. \\ ${ }^{2}$ Hubei Collaborative Innovation Center for Automotive Components Technology, Wuhan University of Technology, Wuhan 430070, China. \\ ${ }^{3}$ SAIC GM Wuling Automobile Co.,Ltd., Liuzhou, 545007, China.
}

\begin{abstract}
The automotive seat headrest plays an important role in the passenger protection during car crashes, and its structure parameters and performance have direct influence on the seat crashworthiness. In this paper, according to relevant regulations of GB11550-2009, collision simulation analyses of the seat headrest structure were carried out by Ls-dyna code. The law of different headrest packaging parameters on collision energy absorption was investigated to provide guidance for the headrest structure optimization design and improvement. The research results show that, with the increase of packaging of the headrest, the maximum acceleration and high acceleration duration time of head gradually decreases.
\end{abstract}

\section{Introduction}

In recent years, along with the increase of car ownership, car rear-end collisions have occurred occasionally. Much attention is paid to the safety of autos. The seat is an important component for ensuring occupant safety and comfort. The headrest is an important part of the seat and plays a key role in the car seat crash safety. During seat headrest design process, a large number of researchers have analyzed safety and comfort of car seat by the dynamic simulation method, especially at the beginning of the seat development stage[1-5]. For optimizing the structure design of seat headrest, a typical seat headrest structure was taken as analysis object and the laws of headrest packaging on collision energy absorption when the steel ball hits the headrest was investigated in this paper.

\section{Model analysis}

A typical seat headrest structure was chosen as the analysis object. According to relevant regulations of GB11550-2009, the steel ball's diameter is $165 \mathrm{~mm}$ and weight is $6.8 \mathrm{~kg}$. At the top of the headrest down along the trunk line with the distance of $65 \mathrm{~mm}$, the steel ball hits the headrest along the horizontal direction at the speed of $\mathrm{V}=22.4 \mathrm{~km} / \mathrm{h}$ to simulate head hits headrest process [6], as shown in Figure 1. The models of headrest frame, seat-back frame were meshed by high accuracy quadrilateral shell elements and headrest structure was meshed by hexahedral elements [7]. The number of shell elements is 35497 and the number of hexahedral elements is 65383.The material of steel ball is rigid body material (*Mat_Rigid). Headrest sponge material is recoverable, low density foam material with hysteresis effect (*Mat_Low_Density_Foam)[8].

Using Surface-to-Surface contact form simulate the crash between rigid ball and seat structure, as shown in Figure 2.

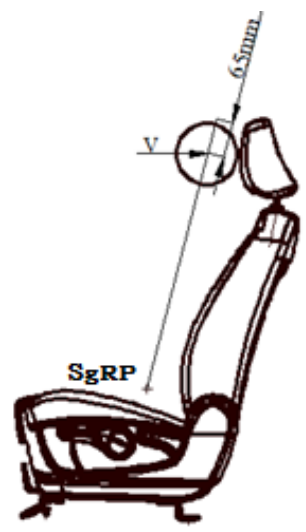

Figure 1. schematic diagram of collision between head and headrest 


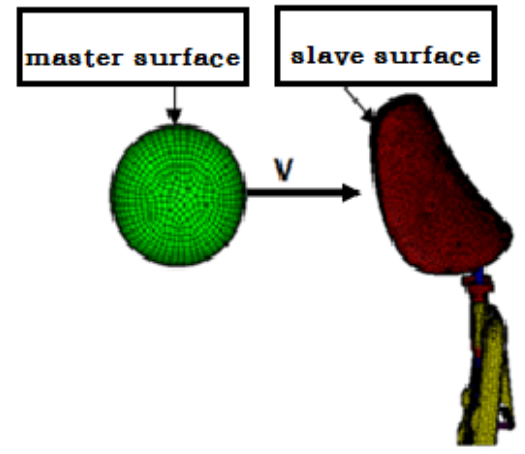

Figure 2. contact surfaces schematic diagram

\section{Collision analysis and discussion}

The main evaluation index for typical seat headrest crash safety in the paper are as follow: 1) the maximum acceleration of the steel ball when the steel ball hits the seat headrest; 2) the duration that the acceleration of the steel ball is more than $25 \mathrm{~g}$ in the collision process (called high acceleration duration in this paper). The maximum acceleration and the duration of the high acceleration of the steel ball are smaller, the headrest's energy absorption and safety, comfort of the occupant are better.

\subsection{Movement process analysis.}

The movement process of the steel ball hitting the seat headrest and the acceleration curve of the steel ball are shown in figure 3, figure 4.During the collision, the ball at the speed of $6.69 \mathrm{~m} / \mathrm{s}$ hits the headrest. In the 2 $\mathrm{ms}$, steel ball contacts with the headrest. Then the steel ball's acceleration gradually increases due to the headrest's buffering effect. With the steel ball continue to move, in the $17 \mathrm{~ms}$, the acceleration reaches to the maximum $34.40 \mathrm{~g}$. With the decrease of the reaction force between the ball and headrest, the acceleration decreases gradually. The steel ball separates from the headrest at $91 \mathrm{~ms}$ and the acceleration of steel ball reduces to about $0 \mathrm{~g}$ and the speed tends to be stable. From the results showing in figure 3 and figure 4, the maximum acceleration of the steel ball hitting the headrest is $34.40 \mathrm{~g}$, which meets regulatory requirements.

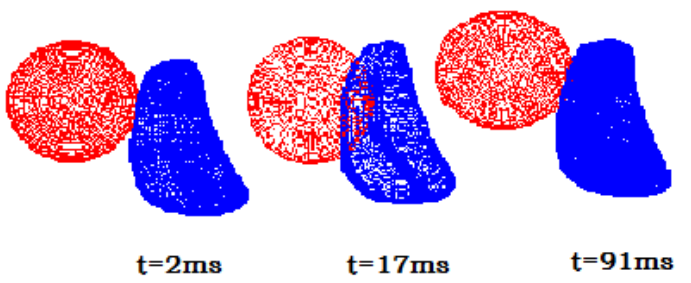

Figure3. steel ball movement diagram

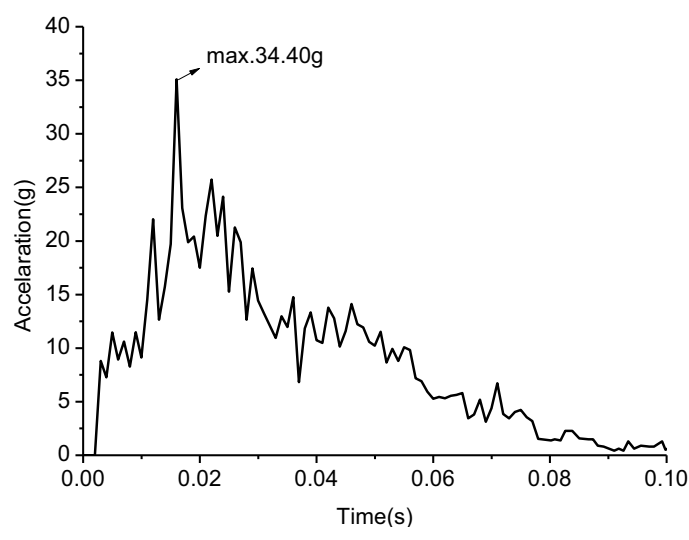

Figure 4. steel ball acceleration-time curve

\subsection{The influence of headrest's packaging on collision energy absorption.}

In the analyses, the headrest's packaging is changed by increasing the thickness of the headrest's both sides with the constant distance of $\mathrm{L}$ (shown as Figure 5). The distance difference B between headrest impact point and headrest both sides thickness represents the headrest's packaging.

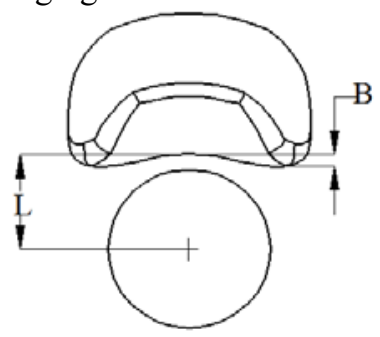

Figure 5. schematic diagram of headrest packaging Under the condition of the headrest's thickness is $28 \mathrm{~mm}$, density is $60 \mathrm{~kg} / \mathrm{m}^{3}$, the headrests which the packaging value $B$ is $6 \mathrm{~mm}, 9 \mathrm{~mm}, 12 \mathrm{~mm}$ respectively are used for collision analysis. The steel ball acceleration curves are shown in figure 6 . With the change of headrest's packaging, the maximum acceleration and high acceleration duration curves are shown in figure 7 . From the figure 7 , it can be found out, the maximum acceleration and high acceleration duration decreases with the increase of the headrest's packaging. This is because contact area between the steel ball and the headrest increases. Per unit area of the steel ball bearing reaction force decreases gradually. The increasing of the headrest's packaging improves the head's lateral stability significantly which can reduce additional head injury during collision [9]. 


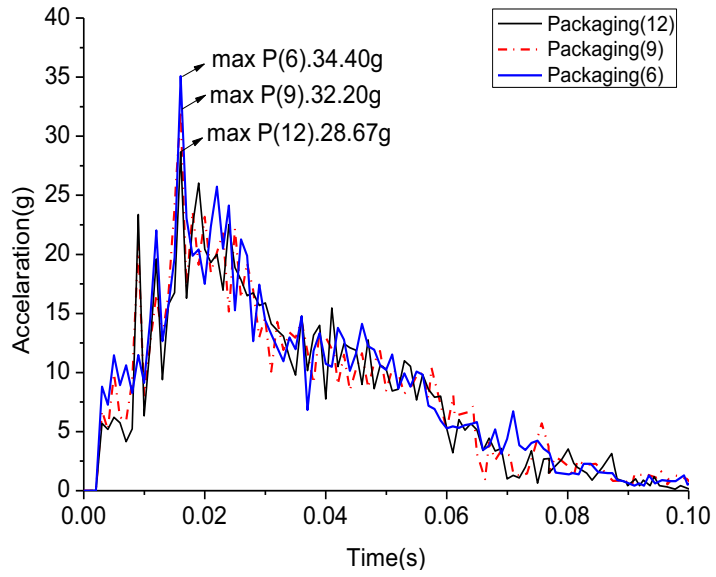

Figure6 steel ball's acceleration curves under the different packaging

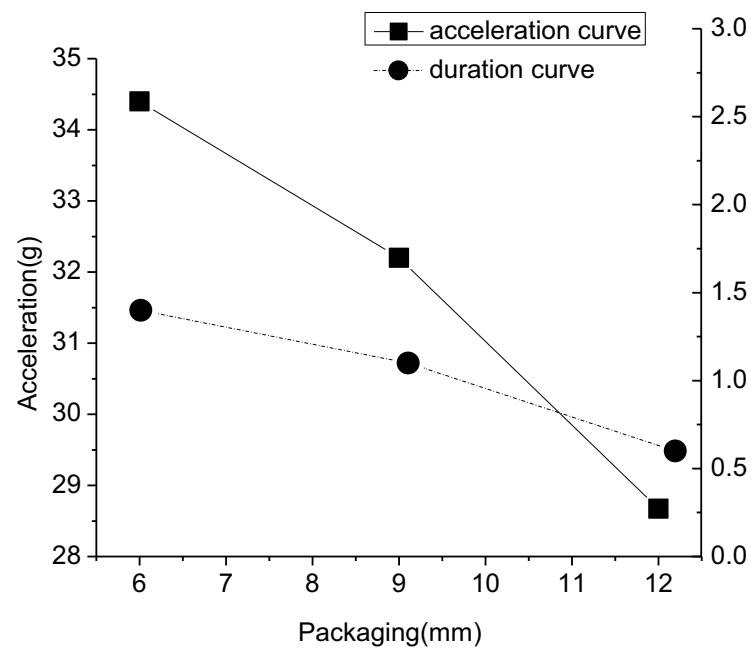

Figure 7 maximum acceleration and high acceleration duration curves

\section{Summary}

In this paper, energy absorption analysis of typical structure's headrest proves that the seat headrest meets regulatory requirement. Besides, the results show that with the increase of the headrest's packaging, head's the maximum acceleration and high acceleration duration of the head decrease gradually, which improve the headrest's energy absorption effectively.

\section{Acknowledgements}

This work was supported by the Natural Science Foundation of Hubei Province of China (No.2014CFB176) and Wuhan Planning Project of Science and Technology (No. 2013011803010606-4).

\section{References}

1. Brix, C. and Tok, C, Robust Design in Occupant Safety Simulation, SAE Int. J. Trans. Safety. 1(2), (2013), 241-260.

2. Fan Pingqing, Song Xinping, Zhao Bo, The analysis on impact capability of seat headrest for automobile, Machinery Design \& Manufacture. 3 (2011), 118-120.

3. Lin Yi, Yao Weimin, Sun Dandan, Study on Automobile Seat Safety Performance During Impact, Journal of Beijing Institute of Technology. 25(1), (2005), 18-20.

4. Jin Jingxu, Song Xuewei, Huang Xuan, Cui Taisong, Effects of combinations of car seat headrest and recliner parameters on whiplash injury, Journal of Harbin Engineering University. 4(10), (2013), 1280-1286.

5. Cao Libo, Li Zhengkun, Gong Yongjian, A Study on the Development and Simulation on an Active Head Restraint, Automotive Engineering. 36(6), (2014), 746-750.

6. GB11550-2009 Strength requirement and test of automobile seats head restraints, Bei Jing Standards Press of China, 2009.

7. Zhang Junyuan, Huang Xuan, Tian Disi, Wang Hongbin, CAE Analysis and Design Modification for Rear Seat Safety During Vehicle Frontal Crash, Automotive Engineering. 33(9), (2011), 767-771.

8. Yao Weimin, Sun Dandan, Parameterization Design of Structure of Automotive Seat Headrest, Automobile Technology. 10 (2004), 4-7.

9. Zou Bowei, The Design of Comfort of Automobile Seat Based on Ergonomics, Automobile Technology. 4 (2009), 15-17. 